\title{
Correspondence
}

\section{Superinfection of perinephric abscess by Stenotrophomonas maltophilia in a tetraplegic patient}

\author{
Spinal Cord (2005) 43, 394-395. doi:10.1038/sj.sc.3101724; Published online 25 January 2005
}

Gram-negative bacteria, present in finished or potable water, can cause infections in hospitalised patients. Stenotrophomonas maltophilia, which is an obligate aerobe, is one of the clinically important organisms present in tap water. The use of tap water in direct patient care and, contamination of distilled water, disinfectants, nebulisers, or ventilator temperature probes present a potential risk of exposure. We report a tetraplegic patient, who developed superinfection of perinephric abscess by $S$. maltophilia.

\section{Case report}

A 50-year-old male with tetraplegia had undergone nephrostomy on 27 September 2003 for pyonephrosis caused by a large calculus in renal pelvis. The urine sample from nephrostomy grew Proteus mirabilis. On 13 October 2003, this patient required insertion of a pigtail catheter to drain a right perinephric abscess. Pus from perinephric abscess also grew Proteus species. The infection was treated with several different antibiotics. Initially teicoplanin, ciprofloxacin, gentamicin and metronidazole were prescribed. After 2 days, antibiotic therapy was changed to ciprofloxacin and cefuroxime, which were continued for another 7 and 4 days, respectively. After an interval of 3 days, ciprofloxacin was again prescribed for 6 days. This was followed by a 5-day course of cefuroxime and gentamicin. After 5 days, he was again prescribed cefuroxime and gentamicin intravenously. He did not receive a carbapenem at any time.

After antibiotic therapy, urine from nephrostomy and drainage fluid from the pigtail catheter located in the perinephric space, sent to the laboratory on 23 October 2003, were sterile. However, S. maltophilia was isolated from a sample of pus, drained from perinephric abscess on 30 October 2003. This organism continued to be isolated in the pus drained from perinephric abscess, as revealed by culture of specimens received for microbiology on 4, 7, 10, 12 and 14 November 2003.

This patient required further treatment with gentamicin in order to control systemic features of sepsis originating from perinephric abscess. Subsequently, a double $\mathbf{J}$ stent was inserted in right ureter. When he became well and the abscess had shrunk to a small size and pus could no longer be aspirated, the pigtail catheter was removed. The stone in the renal pelvis was treated by extracorporeal shock wave lithotripsy.

Initially, this patient was nursed along with tetraplegic subjects, who were ventilator-dependent. When $S$. maltophilia was isolated from the pus, this patient was cohort nursed with three other patients none of whom had tracheostomy or endotracheal tube, and were deemed at low risk for infection with $S$. maltophilia.

We reviewed the infection control measures and ensured that all health professionals, when caring for this patient, followed hand washing, use of gloves and other barrier precautions. We took care to avoid use of indiscriminate and inappropriate antibiotics in the spinal unit especially for urinary infection, ${ }^{1}$ chest infection and pressure sores. Fortunately, S. maltophilia infection was eradicated in this patient and there was no spread of $S$. maltophilia to other patients in the spinal unit.

Possible predisposing factors in this patient for superinfection of perinephric abscess by $S$. maltophilia were indwelling urinary catheter, hospitalisation, administration of multiple courses of broad-spectrum antibiotics, and spinal cord injury. He did not have other predisposing factors such as cancer, neutropenia, bone marrow transplantation, or cytotoxic therapy

In conclusion, this case illustrates the need to review and improve upon infection control measures in spinal injury units, where many patients have indwelling urinary catheters and some patients have tracheostomy. S. maltophilia is usually a nonpathogenic organism or coloniser, but it can cause clinically significant infections of the respiratory and urinary tracts, and soft-tissue infections especially in spinal cord injury patients, as nonspecific and adaptive lymphocytemediated immunity and growth of early haematopoietic progenitor cells are impaired in spinal cord injury patients. $^{2}$

S Vaidyanathan ${ }^{1}$, JA Bowley ${ }^{2}$, BM Soni ${ }^{1}$, JWH Watt ${ }^{1}$, G Singh ${ }^{1}$, PL Hughes ${ }^{3}$ and P Sett ${ }^{1}$

${ }^{1}$ Regional Spinal Injuries Centre, District General Hospital, Southport, Merseyside PR8 6PN, UK; ${ }^{2}$ Department of Microbiology, District General Hospital, Southport, Merseyside PR8 6PN,UK; ${ }^{3}$ Department of Radiology, District General Hospital, Southport, Merseyside PR8 6PN, UK 


\section{References}

1 Vaidyanathan $\mathrm{S}$ et al. Urinary infections in persons with spinal cord injury. Spinal Cord 2004; 42: 659-660.
2 Iversen $\mathrm{PO}$ et al. Depressed immunity and impaired proliferation of haematopoietic progenitor cells in patients with complete spinal cord injury. Blood 2000; 96: 20812083. 